\title{
LMWF5A Demonstrates an Anti-Inflammatory Mode of Action and Similar Drug Targets to Dexamethasone in Activated PBMC
}

Elizabeth D. Frederick

Ampio Pharmaceuticals https://orcid.org/0000-0001-5596-5094

Melissa A. Hausburg

Swedish Medical Center

Gregory W. Thomas

Ampio Pharmaceuticals

David Bar-Or ( $\sim$ davidbme49@gmail.com )

Swedish Medical Center

\section{Research Article}

Keywords: dexamethasone, steroid, anti-inflammatory, osteoarthritis, COVID-19, PBMC

Posted Date: August 11th, 2021

DOI: https://doi.org/10.21203/rs.3.rs-790987/v1

License: (c) (i) This work is licensed under a Creative Commons Attribution 4.0 International License. Read Full License 


\section{Abstract}

The low molecular weight fraction of human serum albumin (LMWF5A) has proven immunomodulatory activity via its effects on multiple inflammatory mediators and transcription factors. This biologic drug is currently being evaluated for the treatment of conditions characterized by hyperactive or persistent immune responses. To gain further insight into the mechanism of action of LMWF5A, an unbiased investigation of its effects on activated immune cells was performed. Peripheral blood mononuclear cells (PBMC) were treated with vehicle control or LMWF5A and stimulated with lipopolysaccharide (LPS), LPS/interferon $y$, or interleukin (IL)-4/IL-13 for 24h. Differential gene expression analysis by RNAseq was performed to determine transcriptional changes due to LMWF5A treatment in each stimulation condition. Ingenuity Pathway Analysis software was employed to reveal overall biological trends observed in the RNAseq datasets and to identify compounds with comparable or contrasting activities to LMWF5A. Analysis of each RNA dataset revealed broad anti-inflammatory and pro-resolving activities for LMWF5A, with predicted inhibition of key pro-inflammatory mediators and functions as well as activation of some anti-inflammatory mediators. Upstream regulator analysis confirmed these activities and identified the corticosteroid dexamethasone as the most significantly similar regulator. Further comparison of LMWF5A and dexamethasone revealed similar, but not identical, targets and directional regulation. This study supports our current knowledge on the mode of action of LMWF5A and provides new hypotheses for future investigations. Due to its analogous biological effects with dexamethasone and known safety profile, LMWF5A may be used to treat conditions that are supported for dexamethasone with fewer or less harmful side effects.

\section{Introduction}

Acute and chronic inflammation-associated diseases are characterized by dysregulated, excessive, and/or sustained immune activation. In general, aberrant activation entails increased expression of proinflammatory proteins, which may arise from enhanced pro-inflammatory transcription factor activity [1, 2]. Previous research has shown that the low molecular weight fraction of human serum albumin (LMWF5A, Ampion ${ }^{\mathrm{TM}}$ ) modulates the levels of cytokines and other inflammatory mediators in various cell models [3]. Much research on the biologic drug LMWF5A has focused on its ability to inhibit release of the pro-inflammatory cytokine tumor necrosis factor a (TNFa) from immune cells, but LMWF5A has also been shown to affect interleukin (IL)-1 $\beta$, IL-6, IL-10, IL-12, interferon (IFN) $\gamma$, chemokine (C-X-C motif) ligand (CXCL) 9, CXCL10, CXCL11, prostaglandin (PG) E2, PGD2, and 15d-PGJ2 due to its impact on regulators upstream of inflammatory protein production [4-10]. Known mechanisms by which LMWF5A exerts its immunomodulatory effects include decreasing pro-inflammatory transcription factor activity (nuclear factor KB [NFKB] and signal transducer and activator of transcription [STAT]) [8], increasing antiinflammatory transcription factor activity (peroxisome proliferator-activated receptor [PPARY] and aryl hydrocarbon receptor [AhR]) [4, 8], and reducing endothelial cell permeability [11]. These modes of action predict that LMWF5A attenuates inflammation, promotes the resolution of the immune response, and restores homeostasis, supporting its use in the treatment of various inflammatory conditions. 
In vitro data on the immunomodulatory activity of LMWF5A are substantiated by its in vivo clinical effects. The efficacy and safety of LMWF5A have been proven in clinical trials for the treatment of the inflammatory condition osteoarthritis $(O A)$ of the knee [12-16]. OA patients, particularly those with severe disease, receiving a single intraarticular injection of LMWF5A experience a statistically significant reduction in pain and improvement in function, with fewer adverse events compared to patients receiving a control injection of saline $[15,16]$. In addition, LMWF5A is currently being assessed for the treatment of coronavirus disease 2019 (COVID-19) complications [17], in which the downstream effects of cytokine storm are observed both in the lung and systemically [18]. Intravenous (IV) injection and nebulization of LMWF5A have been well tolerated, and promising clinical results have been obtained thus far.

Specifically, COVID-19 patients with respiratory distress treated with IV LMWF5A demonstrated stronger clinical improvement as measured by ordinal scale versus standard of care (ClinicalTrials.gov/NCT04456452; unpublished data). The same patient population treated with LMWF5A via inhalation also showed increases in clinical improvement scales as well as reductions in all-cause mortality, hospitalization time, and supplemental oxygen levels versus standard of care (ClinicalTrials.gov/NCT04606784; unpublished data).

Similar to LMWF5A, corticosteroids function by reducing levels of inflammatory mediators and vascular permeability [19]. They have been long and widely used for their potent anti-inflammatory and immunosuppressive properties, and patients with OA or COVID-19, amongst many other conditions, have benefitted from their use. However, the benefits of corticosteroids can be accompanied by adverse events, depending on dosage and duration of use [20]. With respect to intraarticular corticosteroid use for OA, these agents have been reported to induce cartilage cytotoxicity and catabolism, ultimately resulting in disease progression [21]. In COVID-19 patients, oral or IV administration of the corticosteroid dexamethasone has been found to lower mortality rates when disease severity required supplemental oxygen or ventilation $[22,23]$. However, adverse events due to corticosteroid use in this patient population include secondary infections, reactivation of latent infections, avascular necrosis of the hip, hyperglycemia, and psychiatric symptoms [24]. Thus, while corticosteroids are effective in treating these and other inflammatory conditions, their negative side effects highlight the need for safer alternatives.

In this report, an unbiased search for LMWF5A targets and comparable upstream regulators led to the determination that LMWF5A acts on similar, but not identical, targets to dexamethasone. Differences in target number, directional regulation, and identity between LMWF5A and dexamethasone may provide hints regarding the comparatively low incidence of adverse events in patients treated with LMWF5A.

Peripheral blood mononuclear cells (PBMC) were used as an immune cell model, and three different stimulation conditions were applied to evaluate the effect of LMWF5A on cells polarized toward proinflammatory or anti-inflammatory macrophage and lymphocyte phenotypes [25]. RNA sequencing allowed for the determination of differentially expressed genes between LMWF5A and vehicle control. Results were subjected to in silico analysis to 1) reveal overall biological trends observed upon LMWF5A treatment and 2) compare or contrast known upstream regulator activity, i.e., compounds that act in a manner similar or opposite to LMWF5A. Further investigation into the most correlated upstream regulator, 
dexamethasone, was accomplished by evaluating the common downstream targets of this compound and LMWF5A.

\section{Methods}

\section{Cell treatment and RNA isolation}

Frozen human PBMC (ZenBio, Durham, NC, USA) were thawed and resuspended in XVivo 15 medium (Lonza, Verviers, Belgium). $50 \mu \mathrm{L}$ of $2 \times 10^{6}$ cells $/ \mathrm{mL}\left(1 \times 10^{5}\right.$ cells/well final) were treated with $50 \mu \mathrm{L}$ vehicle control (saline; KD Medical, Columbia, MD, USA) or LMWF5A (Ampio Pharmaceuticals, Englewood, CO, USA) in 96-well plates. Cells were then incubated at $37^{\circ} \mathrm{C}$ and $5 \% \mathrm{CO}_{2}$ for $1 \mathrm{~h}$. Immunostimulation was achieved by the addition of $10 \mu \mathrm{L}$ lipopolysaccharide (LPS; final concentration of $100 \mathrm{ng} / \mathrm{mL}$; Sigma, St. Louis, MO, USA), $10 \mu \mathrm{L}$ LPS + IFNY (final concentration of $100 \mathrm{ng} / \mathrm{mL}$ LPS and $20 \mathrm{ng} / \mathrm{mL}$ IFNץ; R\&D Systems, Minneapolis, MN, USA), or $10 \mu \mathrm{L}$ IL-4 + IL-13 (final concentration of 10 $\mathrm{ng} / \mathrm{mL}$ each; R\&D Systems). Cells were further incubated at $37^{\circ} \mathrm{C}$ and $5 \% \mathrm{CO}_{2}$ for $24 \mathrm{~h}$. Cells were pelleted by centrifugation at $1000 \mathrm{rpm}$ for $10 \mathrm{~min}$. Medium was removed, and cells were processed for RNA isolation using the miRNeasy Mini Kit (Qiagen, Hilden, Germany). Ten treatment replicates per plate were combined. This protocol was repeated three times on different days.

\section{RNA sequencing and differential expression analysis}

The resulting RNA samples were subjected to RNAseq by Lexogen (Vienna, Austria). Libraries were manually prepared using the QuantSeq 3' mRNA-Seq FWD Library Prep Kit (Lexogen) following the manufacturer's instructions. Sequencing was performed on a NextSeq 500 System (Illumina, San Diego, CA, USA) using a High Output Kit (v2, 75 cycles; Illumina) in single read mode.

Differential expression between saline and LMWF5A treatments under each stimulation condition was then evaluated. Comparison groups were as follows: LMWF5A + LPS vs. saline + LPS, LMWF5A + LPS/IFNy vs. saline + LPS/IFNy, LMWF5A + IL-4/IL-13 vs. saline + IL-4/IL-13. DESeq2 [26] analysis of raw RNA sequencing counts was performed in $R$ [27] to determine transcript $\log _{2}$ fold-changes, $p$-values, and adjusted $p$-values. Genes with an adjusted $p$-value $<0.05$ were considered differentially expressed.

\section{In silico pathway analysis}

Calculated differential gene expression values were uploaded into Ingenuity Pathway Analysis (IPA) software (Qiagen Digital Insights, Redwood City, CA, USA). An Expression Core Analysis was run based on Expression Log Ratios with an adjusted p-value $<0.05$ using the Ingenuity Knowledge Base as reference. With the default node setting, a graphical summary was generated by IPA that reflected the overall top regulated entities for each individual analysis. Further, this analysis was used to computationally infer upstream regulators that are predicted to have similar or opposite actions to the effect of LMWF5A on the PBMC transcriptome. Z-scores where $z>0$ predicts activation or similar regulation, and $z<0$ predicts the converse; an absolute value of 2 was used as a significance cutoff. Detailed examination of common 
targets between dexamethasone and LMWF5A was accomplished with the 'network' tool in the upstream regulator analysis function with default parameter settings.

\section{Results And Discussion}

\section{Overall biological trends upon LMWF5A treatment}

RNA sequencing results in an extensive, complicated dataset that can be difficult to interpret holistically, highlighting the importance of in silico analysis tools. To understand the overall biological trends present in a dataset of interest, IPA software employs an algorithm to construct a graphical summary that visualizes the most significant canonical pathways, upstream regulators, and biological functions from its core analysis and depicts their relationships to each other.

The RNA sequencing datasets from this study provide a snapshot of all genes that are regulated by LMWF5A in PBMC compared to saline vehicle control under three immunostimulatory conditions after 24 hours of treatment. The number of differentially expressed transcripts (adjusted $p<0.05$ ) were 55, 64, and 139 for the three following comparisons, respectively: LMWF5A + LPS vs. saline + LPS, LMWF5A + LPS/IFNy vs. saline + LPS/IFNy, and LMWF5A + IL-4/IL-13 vs. saline + IL-4/IL-13. The graphical summaries of these datasets present the main biological targets and downstream activities of LMWF5A in this immune cell model. The graphical summary of the LPS/IFNy data is shown as a representative network (Fig. 1); summaries for LPS and IL-4/IL-13 are shown in Supplemental Fig. 1a and b.

Several nodes are common between the three stimulation conditions. All nodes corresponding to biological targets represent molecules that are important in the immune response, such as cytokines, pattern recognition receptors, and inflammation-related transcription factors. Each graphical summary predicts inhibition of multiple interferons (IFNy, IFNa2), interleukins (IL-1 $\beta, I L-6), T N F a$, the hormone and cytokine prolactin (PRL), toll-like receptors (TLRs; TLR7), and transcription factors (interferon regulatory factor [IRF]7, STAT1). In addition, many of the implicated biological functions are associated with a decrease in pro-inflammatory activities. These include the general immune response of cells, cytotoxicity, monocyte/macrophage phagocytosis, integrin-mediated signaling, as well as the development, trafficking, and adhesion of various immune cell types. Furthermore, one pathway demonstrates predicted activation with LMWF5A treatment in IL-4/IL-13-stimulated PBMC, the macrophage-stimulating protein (MSP)- recepteur d'origine nantais (RON) signaling pathway, which has been described as an important negative regulator of inflammation due to its inhibition of pro-inflammatory cytokine production by macrophages [28].

Thus, the overall biological trend observed in PBMC stimulated with LPS, LPS/IFNy, or IL-4/IL-13 in the presence of LMWF5A compared to saline control is an overarching suppression of inflammation, as numerous key pro-inflammatory regulators and functions are predicted to be inhibited and some antiinflammatory regulators are predicted to be activated in this in silico analysis. This drug activity supports 
the use of LMWF5A in a range of clinical conditions that result from a hyperactive or chronic immune response.

\section{Correlation of upstream regulators with LMWF5A}

The identification of molecules that act in a similar or opposite manner to LMWF5A is valuable to further understand how LMWF5A acts on immune cells. The differential gene expression due to LMWF5A treatment in the RNA sequencing datasets was correlated with the activities of known substances using IPA upstream regulator analysis.

Upstream regulator analysis in IPA was utilized to connect LMWF5A-regulated genes with chemicals and proteins that share a subset of targets with analogous biological regulation (Fig. 2a). The significance of the correlation between the molecule in the IPA knowledge base and the uploaded dataset was measured by z-score, with a positive score indicating similar activity to the dataset and a negative score indicating an anti-match in activity. A comparison analysis of upstream regulators was performed to visualize regulators that are relevant to all stimulation conditions simultaneously. Figure $2 b$ lists the top upstream regulators that correlate with LMWF5A-regulated transcripts, while Fig. $2 \mathrm{c}$ lists the top regulators that are inversely correlated.

Interestingly, the upstream regulator with the highest total z-score was the corticosteroid dexamethasone (Fig. 2b), which acts as a glucocorticoid receptor agonist and affects a multitude of indirect downstream targets [29]. The primary action of dexamethasone is to suppress inflammatory cell activity and inflammatory mediator levels, which mirrors the biological trends predicted by IPA for LMWF5A in this study as well as previously published work on LMWF5A. Dexamethasone, as a synthetic steroid, has multiple indications, including inflammatory conditions (such as rheumatoid and psoriatic arthritis, systemic lupus erythematosus, and Crohn's disease), multiple sclerosis, cerebral edema, shock, and allergies, amongst others [30]. Importantly, dexamethasone has also been administered as an intraarticular injection for joint inflammation [30] and has been recently studied as a potent anti-inflammatory for the treatment of COVID-19 [22]; both conditions are currently being investigated in LMWF5A clinical trials.

The other top five most positively correlated upstream regulators with LMWF5A (Fig. 2b) include sirtuin 1 (SIRT1), prostaglandin E receptor 4 (PTGER4), filgrastim, and SB203580. Like dexamethasone, each of these compounds has demonstrated anti-inflammatory activity.

SIRT1 is a NAD-dependent protein deacetylase that negatively regulates inflammation by altering cytokine levels and immune cell recruitment and activation by deacetylating and suppressing transcription factors, including NFKB [31]. Similarly, LMWF5A has also been proven to modulate transcription factor activity to decrease pro-inflammatory cytokine levels. Its ability to reduce the release of both TNFa and IL-1 $\beta$ has been linked to its effects on the NFKB-repressing PPARY and AhR pathways in LPS-stimulated PBMC, and it has also been shown to prevent NFKB reporter activity [8]. 
PTGER4 (or EP4) is a transmembrane, G-coupled protein receptor that becomes activated when bound to the cyclooxygenase (COX) pathway product PGE2, which is induced during inflammation. Although the role of PGE2 is pleotropic, it exerts its anti-inflammatory effects via PTGER4. PTGER4 binds to EP4 receptor-associated protein, which in turn, reduces the phosphorylation and increases the stability of p105, an inhibitor of NFKB and mitogen-activated protein/extracellular signal-regulated kinase (MEK) [32]. Overall, PTGER4 downregulates inflammation by modulating macrophage cytokine and chemokine secretion as well as T cell proliferation, differentiation, and cytokine production [33]. LMWF5A has been associated with an upregulation of the COX pathway and its products, including the PTGER4 ligand PGE2, in PBMC and primary human osteoarthritic cells $[7,9]$. With respect to the COX pathway, the activity of LMWF5A may be unique to the anti-inflammatory drug class, as most inhibit both cytokine and prostaglandin release while LMWF5A inhibits cytokine release but promotes prostaglandin release. Inhibition of the COX/prostaglandin pathway can result in harmful side effects [34], and the stimulation of this pathway with concomitant inhibition of cytokine production by LMWF5A may, conversely, promote anti-inflammation, resolution, and healing.

Filgrastim, recombinant human granulocyte colony stimulating factor, is commonly used clinically as a complement to chemotherapy due to its ability to stimulate granulocyte production, thus preventing low white blood cell counts [35]. In addition, this protein has confirmed anti-inflammatory properties in vivo with respect to the cytokine response and has been suggested as a potential treatment for chronic inflammatory conditions; for example, LPS-induced cytokine release has been shown to be attenuated in healthy human volunteers treated with filgrastim $[36,37]$.

Finally, SB203580 is a potent p38 mitogen-activated protein kinase (MAPK) inhibitor, with strong effects on cytokine production [38]. The p38 MAPK signaling pathway is critical to the regulation of many cellular processes, including inflammation, as it is activated in response to inflammatory mediators and other stress-related molecules and acts as a major regulator of cytokine production [39]. Overall, the top upstream regulators that significantly match LMWF5A activity have been empirically proven to downregulate components critical to inflammation, including pro-inflammatory cytokines and transcription factors.

In contrast, the top five upstream regulators that do not match the actions of LMWF5A and have the lowest total z-scores are lipopolysaccharide (LPS), IFNy, STAT1, poly rl:rC-RNA, and IFNa2 (Fig. 2c). This list consists of established pro-inflammatory pathogen- and damage-associated molecular patterns (P/DAMP), transcription factors, and cytokines. During the inflammatory cascade, recognition of PAMPs and DAMPs by pattern recognition receptors, like TLRs, results in signal transduction to turn on multiple transcription factors (NF-KB, MAPK, STAT) that increase the expression of key cytokines that promote inflammation [1].

LPS and poly rl:rC-RNA are both categorized as PAMPs and function as signals of infection to initiate immune signaling via TLRs. LPS is a major component of Gram-negative bacterial cell walls. It acts as a PAMP upon bacterial infection in vivo and is widely utilized in vitro to stimulate the TLR4-induced 
immune response, which can occur upon recognition of either pathogens or endogenous molecules that are released upon tissue damage [40]. LPS was used in two of the three conditions in this study to stimulate an immune response in $\mathrm{PBMC}$, and its ranking as the most negatively correlated upstream regulator to LMWF5A highlights the fact that LMWF5A strongly counteracts the inflammatory outcomes of TLR4 signaling. Moreover, the anti-inflammatory effects of LMWF5A on cytokine release and transcription factor activity have been extensively studied using cells treated with LPS as a TLR4 stimulant $[4,8,41]$. poly rl:rC-RNA is a dsRNA mimic that can simulate infection with a dsRNA virus and activate TLR3 [42], suggesting that LMWF5A may also offset the actions of other TLR-driven pathways; the relationships between LMWF5A and other TLRs are currently under investigation.

As additional opposing upstream regulators, the cytokines IFNY and IFNa2 represent both classes of interferons, type II and I, respectively. They are secreted upon viral infection to limit viral replication and regulate the subsequent immune response [43]. The identification of these IFNs as highly ranked, negatively correlated upstream regulators to LMWF5A emphasizes the potential benefits of LMWF5A for the treatment of viral infections that involve a hyperimmune response or cytokine storm, including COVID19 [44]. The Janus kinase (JAK)-STAT signaling pathway is the most studied IFN-related transcription factor pathway, but IFNs also activate other signaling cascades, including p38 MAPK and phosphatidylinositol 3'-kinase, to exert their anti-viral and pro-inflammatory effects [45].

Because both type I and II IFNs activate STAT complexes, it is not surprising that STAT1 is also part of this list of opposing upstream regulators. Upon recognition of IFNs, a variety of interleukins, or other cytokines, STAT1 is phosphorylated, mainly by JAK kinases, and activated to drive a pro-inflammatory cascade [46]. LMWF5A was also previously demonstrated to inhibit the ability of STAT1 to bind its cognate DNA sequence in LPS-stimulated PBMC, implicating regulation of this transcription factor as part of the LMWF5A mode of action [8]. Inhibition of the JAK-STAT pathway has been suggested as a therapeutic strategy for the treatment of inflammatory conditions, including rheumatoid arthritis (RA), psoriasis, inflammatory bowel disease [47] as well as COVID-19 [48]. In summary, the analysis of inversely correlated upstream regulators of LMWF5A represent pro-inflammatory factors, emphasizing the anti-inflammatory activity of LMWF5A.

\section{Comparison of dexamethasone and LMWF5A targets}

In the three different immunostimulatory conditions used to evaluate the effect of LMWF5A on PBMC, dexamethasone was determined to be the most significant positively correlated upstream regulator to LWMF5A. Due to this finding and the longstanding and widespread utility of dexamethasone in the clinic, a detailed comparison of its targets to the LMWF5A datasets was performed.

The IPA Ingenuity Knowledge Base is an extensive database that is comprised of empirically proven interactions and relationships between genes, proteins, drugs, etc. By querying the Knowledge Base for factors both linked to dexamethasone and differentially expressed upon LMWF5A treatment, it was determined that LMWF5A regulates 29, 26, and 42 established dexamethasone targets for the LPS/IFNy, LPS, and IL-4/IL-13 stimulation conditions, respectively. These common targets represent a large 
proportion of each LMWF5A dataset, with $53 \%, 41 \%$, and $30 \%$ of the transcripts significantly regulated by LMWF5A are also targeted by dexamethasone. However, based on IPA computational modeling, directional regulation by LMWF5A of 7, 10, and 17 of the common targets were inconsistent with that of dexamethasone for the LPS/IFNy, LPS, and IL-4/IL-13 stimulation conditions, respectively. Nevertheless, overall IPA modeling predicted significantly similar regulation of downstream molecules by dexamethasone and LMWF5A based on directionally correlated transcripts. The common targets between dexamethasone and LMWF5A are shown for the LPS/IFNY stimulation conditions in a representative figure (Fig. 3). The common targets for the LPS and IL-4/IL-13 conditions are presented in Supplemental Fig. 2a and b.

In Fig. 3, 29 transcripts are presented that were regulated by LMWF5A in LPS/IFNy-stimulated PBMC at $24 \mathrm{~h}$ that are also known targets of dexamethasone. Of these transcripts, 26 were decreased by LMWF5A (green) and 3 were increased (red). Further, of the 29 transcripts, 19 were directionally consistent with the action of dexamethasone (orange or blue lines) and 7 were directionally inconsistent (yellow lines); 3 dexamethasone targets have unpredicted directional regulation (gray lines).

An example of a target that is regulated by LMWF5A in a direction consistent with dexamethasone is $\mathrm{C}-\mathrm{C}$ motif chemokine ligand 2 (CCL2), also called monocyte chemoattractant-1. Upon recognition of inflammatory stimuli, CCL2 expression is induced, and this chemokine drives migration of immune cells, particularly monocytes, to the site of infection or tissue injury [49]. Dysregulated, increased CCL2 expression is linked to the pathology of many diseases, including heart failure, RA, and diabetes, due its overpromotion of immune cell infiltration and downstream pro-inflammatory effects [50]. Serum CCL2 has been demonstrated to be increased in OA patients versus healthy controls, suggesting its importance to OA pathogenesis [51]. Some studies have reported increased CCL2 levels in OA synovial fluid as well, and CCL2 has been linked to OA-associated pain in addition to factors influencing cartilage catabolism [52]. With respect to COVID-19, CCL2, along with many other inflammatory cytokines, contributes to the cytokine storm that occurs during the body's dysregulated response to SARS-CoV2 infection and its level has been correlated with increased disease severity [48]. Thus, the similar activities of both dexamethasone and LMWF5A on CCL2 should provide benefit to patients with CCL2-related diseases, including OA and COVID-19.

Conversely, an example of a target that is regulated by LMWF5A in a direction inconsistent with dexamethasone is cathepsin B (CTSB). CTSB is member of the cathepsin family of cysteine proteases, which are localized in the lysosome [53]. It is well studied in the context of cancer [54] but has also been implicated in cartilage degradation and OA pathogenesis due to its proteolytic activity of extracellular matrix components and its ability to promote the activity of other proteases [55]. In fact, the enzymatic activity of CTSB was demonstrated to be enhanced in vitro in stimulated primary chondrocytes and in vivo in cartilage, serum, and synovial fluid from OA patients, in which CTSB activity levels were associated with OA disease severity [56]. In the IPA upstream regulator analysis, the Knowledge Database records CTSB as being upregulated by dexamethasone, supporting the cartilage-degrading effects observed with corticosteroids, while the differential expression analysis showed that CTSB was 
downregulated by LMWF5A. This and other differences may be important distinctions between these two anti-inflammatory treatments. While it is possible to explore each specific directionally consistent or inconsistent target, the present in silico analysis allows for a broad understanding of overall LMWF5A activity and its comparison to dexamethasone as well as the interplay between common targets and their downstream pathways.

Further comparison of dexamethasone and LMWF5A revealed 71 total common targets (Fig. 4). Of the 71 common targets, only five transcripts were found to be affected by LMWF5A under all three stimulation conditions: $\beta$-actin (ACTB), $\gamma$-actin (ACTG1), myristoylated alanine-rich C-kinase substrate (MARCKS), and tryptophanyl-tRNA synthetase 1 (WARS1), all associated with the cytoskeleton, and cytochrome b-245 b chain (CYBB; also called NADPH oxidase 2), which functions to produce reactive oxygen species to eliminate pathogens during infection and is linked to the development of acute respiratory distress syndrome $[57,58]$. Ten of the targets were shared between the datasets containing LPS, and three were shared between the IL-4/IL-13 and each of the LPS and LPS/IFNy datasets. Moreover, 50 of the 71 common targets were unique to one stimulation condition, highlighting the wide-ranging effects of LMWF5A as well as its different activities depending on the inflammatory milieu. This result suggests that LMWF5A treatment may be beneficial in multiple inflammatory environments.

The differences in common targets between the datasets points to a limitation of the current study. This analysis solely examined the effects of LMWF5A in one cell model at one time point, albeit with three immunostimulation conditions. Additional timepoints or cell types would provide even further insight into the mode of action of LMWF5A and may identify more overlapping targets. For example, due to the known effects of LMWF5A on cytokine release from PBMC, an earlier timepoint may have captured more differentially expressed cytokine RNAs as well as any temporal differences in cytokine RNA levels due to LMWF5A treatment. However, extensive information has been gained with the large datasets in this investigation.

\section{Conclusion}

Unbiased in silico analysis of RNA sequencing datasets from activated PBMC treated with the biologic drug LMWF5A compared to vehicle control demonstrates that LMWF5A targets numerous inflammationrelated factors and functions, ultimately resulting in a broad suppression of inflammation and a putative promotion of inflammatory resolution. Upstream regulator analysis identified dexamethasone as the most positively correlated molecule to LMWF5A. Due to significant overlap between their targets, LMWF5A may be indicated for the many conditions in which dexamethasone is used. This corticosteroid and LMWF5A exhibit extensive anti-inflammatory effects due to their ability to simultaneously inhibit multiple cytokines by affecting upstream activity. This differs from other treatments of acute and chronic inflammatory conditions that inhibit individual cytokines, such as antibody-based medicines. In addition, dexamethasone targets many molecules that are not regulated by LMWF5A, and some of the targets that are regulated by both are inversely affected. These observations may help to explain why dexamethasone is associated with side-effects that are not experienced by LMWF5A-treated patients. Furthermore, this 
study supports our previous research and provides new hypotheses for future investigations on the mode of action of LMWF5A.

\section{Declarations}

\section{Funding}

Funding for this work was provided by Ampio Pharmaceuticals.

\section{Conflicts of interest}

E.D.F. is an employee and has been granted stock options at Ampio Pharmaceuticals. M.A.H. is a consultant, shareholder, and has been granted stock options at Ampio Pharmaceuticals. G.W.T. is an employee, shareholder, and has been granted stock options at Ampio Pharmaceuticals. D.B-O. is a member of the board of directors and shareholder at Ampio Pharmaceuticals.

\section{Availability of data and material}

The datasets generated and analyzed during the current study are available from the corresponding author upon request. Full datasets will be uploaded to Gene Expression Omnibus upon manuscript acceptance.

\section{Code availability}

Code is available from the corresponding author upon request.

\section{Author contributions}

E.D.F.: Conception; experimental design and execution; data acquisition, analysis, and interpretation; drafting of manuscript. M.A.H.: Conception; data acquisition, analysis, and interpretation; drafting of manuscript. G.W.T.: Critical review and revision of the manuscript. D.B-O.: Conception; project supervision; data interpretation; critical review and revision of the manuscript. All authors have read and approved the manuscript.

\section{Ethics approval and consent to participate}

All experiments were conducted with commercially available primary human cells, with all appropriate consents available from the supplier.

\section{Consent for publication}

Not applicable.

\section{Acknowledgements}


The authors acknowledge technical support from Lexogen $\mathrm{GmbH}$ for library preparation and sequencing.

\section{References}

1. Chen, L., H. Deng, H. Cui, J. Fang, Z. Zuo, and J. Deng, et al. 2018. Inflammatory responses and inflammation-associated diseases in organs. Oncotarget 9 (6): 7204-7218. https://doi.org/10.18632/oncotarget.23208.

2. Medzhitov, R., and T. Horng. 2009. Transcriptional control of the inflammatory response. Nat Rev Immunol. 9 (10): 692-703. https://doi.org/10.1038/nri2634.

3. Bar-Or, D., G. Thomas, L. T. Rael, E. Frederick, M. Hausburg, and R. Bar-Or, et al. 2019. On the Mechanisms of Action of the Low Molecular Weight Fraction of Commercial Human Serum Albumin in Osteoarthritis. Curr Rheumatol Rev 15 (3): 189-200. https://doi.org/10.2174/1573397114666181119121519.

4. Rael, L. T., R. Bar-Or, K. L. Banton, C. W. Mains, M. Roshon, and A. H. Tanner, et al. 2018. The antiinflammatory effect of LMWF5A and N-acetyl kynurenine on macrophages: Involvement of aryl hydrocarbon receptor in mechanism of action. Biochem Biophys Rep 15: 61-67. https://doi.org/10.1016/j.bbrep.2018.06.006.

5. Shimonkevitz, R., G. Thomas, D. S. Slone, M. Craun, C. Mains, and D. Bar-Or. 2008. A diketopiperazine fragment of human serum albumin modulates T-lymphocyte cytokine production through rap1. $J$ Trauma 64 (1): 35-41. https://doi.org/10.1097/TA.0b013e3181589ff9.

6. Bar-Or, D., G. W. Thomas, R. Bar-Or, L. T. Rael, K. Scarborough, and N. Rao, et al. 2006. Commercial human albumin preparations for clinical use are immunosuppressive in vitro. Crit Care Med 34 (6): 1707-1712. https://doi.org/10.1097/01.CCM.0000217923.53680.4C.

7. Frederick, E. D., M. A. Hausburg, G. W. Thomas, L. T. Rael, E. Brody, and D. Bar-Or. 2016. The low molecular weight fraction of human serum albumin upregulates $C O X 2$, prostaglandin $E 2$, and prostaglandin D2 under inflammatory conditions in osteoarthritic knee synovial fibroblasts. Biochem Biophys Rep 8: 68-74. https://doi.org/10.1016/j.bbrep.2016.08.015.

8. Thomas, G., E. Frederick, L. Thompson, R. Bar-Or, Y. Mulugeta, and M. Hausburg, et al. 2020. LMWF5A suppresses cytokine release by modulating select inflammatory transcription factor activity in stimulated PBMC. J Trans/ Med 18 (1): 452. https://doi.org/10.1186/s12967-020-02626-z.

9. Thomas, G. W., L. T. Rael, M. Hausburg, E. D. Frederick, C. W. Mains, and D. Slone, et al. 2016. The low molecular weight fraction of human serum albumin upregulates production of $15 \mathrm{~d}-\mathrm{PGJ} 2$ in Peripheral Blood Mononuclear Cells. Biochem Biophys Res Commun 473 (4): 1328-1333. https://doi.org/10.1016/j.bbrc.2016.04.072.

10. Hausburg, M. A., E. D. Frederick, P. McNair, J. Schwappach, K. L. Banton, and M. Roshon, et al. 2018. Clinically relevant redifferentiation of fibroblast-like chondrocytes into functional chondrocytes by the low molecular weight fraction of human serum albumin. Clin Exp Rheumato/ 36 (5): 891-895. 
11. Thomas, G. W., L. T. Rael, M. Hausburg, E. D. Frederick, E. Brody, and D. Bar-Or. 2016. The low molecular weight fraction of commercial human serum albumin induces acetylation of alpha-tubulin and reduces transcytosis in retinal endothelial cells. Biochem Biophys Res Commun 478 (4): 17801785. https://doi.org/10.1016/j.bbrc.2016.09.026.

12. Bar-Or, D., K. M. Salottolo, H. Loose, M. J. Phillips, B. McGrath, and N. Wei, et al. 2014. A randomized clinical trial to evaluate two doses of an intra-articular injection of LMWF-5A in adults with pain due to osteoarthritis of the knee. PLoS One. 9 (2): e87910. https://doi.org/10.1371/journal.pone.0087910.

13. McGrath, B. 2015. Unique aspects of pain reduction in osteoarthritis of the knee with LMWF-5A. Open Access Rheumatol 7: 19-22. https://doi.org/10.2147/OARRR.S71537.

14. Schwappach, J., S. M. Dryden, and K. M. Salottolo. 2017. Preliminary Trial of Intra-articular LMWF-5A for Osteoarthritis of the Knee. Orthopedics. 40 (1): e49-e53. https://doi.org/10.3928/0147744720160926-02.

15. Cole, B., B. McGrath, K. Salottolo, and D. Bar-Or. 2018. LMWF-5A for the Treatment of Severe Osteoarthritis of the Knee: Integrated Analysis of Safety and Efficacy. Orthopedics. 41 (1): e77-e83. https://doi.org/10.3928/01477447-20171114-05.

16. Salottolo, K., B. Cole, and D. Bar-Or. 2018. Intra-articular injection of the anti-inflammatory compound LMWF-5A in adults with severe osteoarthritis: a double-blind prospective randomized controlled multi-center safety and efficacy trial. Patient Saf Surg 12: 11. https://doi.org/10.1186/s13037-0180158-0.

17. Thomas, G., E. Frederick, M. Hausburg, L. Goldberg, M. Hoke, and M. Roshon, et al. 2020. The novel immunomodulatory biologic LMWF5A for pharmacological attenuation of the "cytokine storm" in COVID-19 patients: a hypothesis. Patient Saf Surg 14: 21. https://doi.org/10.1186/s13037-02000248-4.

18. Tang, L., Z. Yin, Y. Hu, and H. Mei. 2020. Controlling Cytokine Storm Is Vital in COVID-19. Front Immuno/ 11: 570993. https://doi.org/10.3389/fimmu.2020.570993.

19. Barnes, P. J. 2006. How corticosteroids control inflammation: Quintiles Prize Lecture 2005. Br J Pharmacol 148 (3): 245-254. https://doi.org/10.1038/sj.bjp.0706736.

20. Yasir, M., A. Goyal, P. Bansal, and S. Sonthalia. Corticosteroid Adverse Effects. StatPearls. Treasure Island (FL)2021.

21. Wernecke, C., H. J. Braun, and J. L. Dragoo. 2015. The Effect of Intra-articular Corticosteroids on Articular Cartilage: A Systematic Review. Orthop J Sports Med 3 (5): 2325967115581163. https://doi.org/10.1177/2325967115581163.

22. Ahmed, M. H., and A. Hassan. 2020. Dexamethasone for the Treatment of Coronavirus Disease (COVID-19): a Review. SN Compr Clin Med.1-10. https://doi.org/10.1007/s42399-020-00610-8.

23. Group, R. C., P. Horby, W. S. Lim, J. R. Emberson, M. Mafham, and J. L. Bell, et al. 2021. Dexamethasone in Hospitalized Patients with Covid-19. N Engl J Med 384 (8): 693-704. https://doi.org/10.1056/NEJMoa2021436. 
24. Chen, F., L. Hao, S. Zhu, X. Yang, W. Shi, and K. Zheng, et al. 2021. Potential Adverse Effects of Dexamethasone Therapy on COVID-19 Patients: Review and Recommendations. Infect Dis Ther. https://doi.org/10.1007/s40121-021-00500-z. .1-25.

25. Martinez, F. O., A. Sica, A. Mantovani, and M. Locati. 2008. Macrophage activation and polarization. Front Biosci 13: 453-461. https://doi.org/10.2741/2692.

26. Love, M. I., W. Huber, and S. Anders. 2014. Moderated estimation of fold change and dispersion for RNA-seq data with DESeq2. Genome Bio/ 15 (12): 550. https://doi.org/10.1186/s13059-014-0550-8.

27. $\mathrm{R}$ Core Team. 2021. R: A language and environment for statistical computing. Vienna, Austria: $\mathrm{R}$ Foundation for Statistical Computing.

28. Huang, L., X. Fang, D. Shi, S. Yao, W. Wu, and Q. Fang, et al. 2020. MSP-RON Pathway: Potential Regulator of Inflammation and Innate Immunity. Front Immunol 11: 569082. https://doi.org/10.3389/fimmu.2020.569082.

29. Newton, R. 2000. Molecular mechanisms of glucocorticoid action: what is important? Thorax. 55(7):603 - 13. https://doi.org/10.1136/thorax.55.7.603.

30. Lexicomp. n.d. Dexamethasone. https://www.uptodate.com/contents/dexamethasone-systemicdrug-information\#references. Accessed 05 August 2021.

31. Vachharajani, V. T., T. Liu, X. Wang, J. J. Hoth, B. K. Yoza, and C. E. McCall. 2016. Sirtuins Link Inflammation and Metabolism. J Immunol Res 2016: 8167273. https://doi.org/10.1155/2016/8167273.

32. Minami, M., K. Shimizu, Y. Okamoto, E. Folco, M. L. llasaca, and M. W. Feinberg, et al. 2008. Prostaglandin E receptor type 4-associated protein interacts directly with NF-kappaB1 and attenuates macrophage activation. J Biol Chem 283 (15): 9692-9703. https://doi.org/10.1074/jbc.M709663200.

33. Tang, E. H., P. Libby, P. M. Vanhoutte, and A. Xu. 2012. Anti-inflammation therapy by activation of prostaglandin EP4 receptor in cardiovascular and other inflammatory diseases. $J$ Cardiovasc Pharmacol. 59 (2): 116-123. https://doi.org/10.1097/FJC.0b013e3182244a12.

34. Ong, C. K., P. Lirk, C. H. Tan, and R. A. Seymour. 2007. An evidence-based update on nonsteroidal antiinflammatory drugs. Clin Med Res 5 (1): 19-34. https://doi.org/10.3121/cmr.2007.698.

35. Frampton, J. E., C. R. Lee, and D. Faulds. 1994. Filgrastim. A review of its pharmacological properties and therapeutic efficacy in neutropenia. Drugs. 48 (5): 731-760. https://doi.org/10.2165/00003495$199448050-00007$.

36. Hartung, T. 1999. Anti-inflammatory aspects of Filgrastim and impact on IL-2 release. J Hematother Stem Cell Res 8 (Suppl 1): S21-S22. https://doi.org/10.1089/152581699319885.

37. Hartung, T., W. D. Doecke, D. Bundschuh, M. A. Foote, F. Gantner, and C. Hermann, et al. 1999. Effect of filgrastim treatment on inflammatory cytokines and lymphocyte functions. Clin Pharmacol Ther 66 (4): 415-424. https://doi.org/10.1053/cp.1999.v66.a101210.

38. Cuenda, A., J. Rouse, Y. N. Doza, R. Meier, P. Cohen, and T. F. Gallagher, et al. 1995. SB 203580 is a specific inhibitor of a MAP kinase homologue which is stimulated by cellular stresses and 
interleukin-1. FEBS Lett 364 (2): 229-233. https://doi.org/10.1016/0014-5793(95)00357-f.

39. Yang, Y., S. C. Kim, T. Yu, Y. S. Yi, M. H. Rhee, and G. H. Sung, et al. 2014. Functional roles of p38 mitogen-activated protein kinase in macrophage-mediated inflammatory responses. Mediators Inflamm. 2014:352371. https://doi.org/10.1155/2014/352371.

40. Lu, Y. C., W. C. Yeh, and P. S. Ohashi. 2008. LPS/TLR4 signal transduction pathway. Cytokine 42 (2): 145-151. https://doi.org/10.1016/j.cyto.2008.01.006.

41. Thomas, G. W., L. T. Rael, C. W. Mains, D. Slone, M. M. Carrick, and R. Bar-Or, et al. 2016. AntiInflammatory Activity in the Low Molecular Weight Fraction of Commercial Human Serum Albumin (LMWF5A). J Immunoassay Immunochem 37 (1): 55-67.

https://doi.org/10.1080/15321819.2015.1047516.

42. Alexopoulou, L., A. C. Holt, R. Medzhitov, and R. A. Flavell. 2001. Recognition of double-stranded RNA and activation of NF-kappaB by Toll-like receptor 3. Nature 413 (6857): 732-738. https://doi.org/10.1038/35099560.

43. Lee, A. J., and A. A. Ashkar. 2018. The Dual Nature of Type I and Type II Interferons. Front Immuno/ 9 : 2061. https://doi.org/10.3389/fimmu.2018.02061.

44. Nile, S. H., A. Nile, J. Qiu, L. Li, X. Jia, and G. Kai. 2020. COVID-19: Pathogenesis, cytokine storm and therapeutic potential of interferons. Cytokine Growth Factor Rev 53: 66-70. https://doi.org/10.1016/j.cytogfr.2020.05.002.

45. Platanias, L. C. 2005. Mechanisms of type-l- and type-II-interferon-mediated signalling. Nat Rev Immuno/ 5 (5): 375-386. https://doi.org/10.1038/nri1604.

46. Ramana, C. V., M. Chatterjee-Kishore, H. Nguyen, and G. R. Stark. 2000. Complex roles of Stat1 in regulating gene expression. Oncogene. 19 (21): 2619-2627.

https://doi.org/10.1038/sj.onc.1203525.

47. Banerjee, S., A. Biehl, M. Gadina, S. Hasni, and D. M. Schwartz. 2017. JAK-STAT Signaling as a Target for Inflammatory and Autoimmune Diseases: Current and Future Prospects. Drugs. 77 (5): 521-546. https://doi.org/10.1007/s40265-017-0701-9.

48. Chen, Y., J. Wang, C. Liu, L. Su, D. Zhang, and J. Fan, et al. 2020. IP-10 and MCP-1 as biomarkers associated with disease severity of COVID-19. Mol Med 26 (1): 97. https://doi.org/10.1186/s10020020-00230-x.

49. Deshmane, S. L., S. Kremlev, S. Amini, and B. E. Sawaya. 2009. Monocyte chemoattractant protein-1 (MCP-1): an overview. J Interferon Cytokine Res 29 (6): 313-326. https://doi.org/10.1089/jir.2008.0027.

50. Gschwandtner, M., R. Derler, and K. S. Midwood. 2019. More Than Just Attractive: How CCL2 Influences Myeloid Cell Behavior Beyond Chemotaxis. Front Immunol 10: 2759. https://doi.org/10.3389/fimmu.2019.02759.

51. Ni, F., Y. Zhang, X. Peng, and J. Li. 2020. Correlation between osteoarthritis and monocyte chemotactic protein-1 expression: a meta-analysis. J Orthop Surg Res 15 (1): 516. https://doi.org/10.1186/s13018-020-02045-2. 
52. Scanzello, C. R. 2017. Chemokines and inflammation in osteoarthritis: Insights from patients and animal models. J Orthop Res 35 (4): 735-739. https://doi.org/10.1002/jor.23471.

53. Turk, V., V. Stoka, O. Vasiljeva, M. Renko, T. Sun, and B. Turk, et al. 2012. Cysteine cathepsins: from structure, function and regulation to new frontiers. Biochim Biophys Acta 1824 (1): 68-88. https://doi.org/10.1016/j.bbapap.2011.10.002.

54. Aggarwal, N., and B. F. Sloane. 2014. Cathepsin B: multiple roles in cancer. Proteomics Clin Appl. 8 (5-6): 427-437. https://doi.org/10.1002/prca.201300105.

55. Baici, A., A. Lang, R. Zwicky, and K. Muntener. 2005. Cathepsin B in osteoarthritis: uncontrolled proteolysis in the wrong place. Semin Arthritis Rheum 34 (6 Suppl 2): 24-28. https://doi.org/10.1016/j.semarthrit.2004.03.008.

56. Ben-Aderet, L., E. Merquiol, D. Fahham, A. Kumar, E. Reich, and Y. Ben-Nun, et al. 2015. Detecting cathepsin activity in human osteoarthritis via activity-based probes. Arthritis Res Ther 17: 69. https://doi.org/10.1186/s13075-015-0586-5.

57. Carnesecchi, S., J. C. Pache, and C. Barazzone-Argiroffo. 2012. NOX enzymes: potential target for the treatment of acute lung injury. Cell Mol Life Sci 69 (14): 2373-2385. https://doi.org/10.1007/s00018-012-1013-6.

58. Diebold, B. A., S. M. Smith, Y. Li, and J. D. Lambeth. 2015. NOX2 As a Target for Drug Development: Indications, Possible Complications, and Progress. Antioxid Redox Signal 23 (5): 375-405. https://doi.org/10.1089/ars.2014.5862.

\section{Figures}




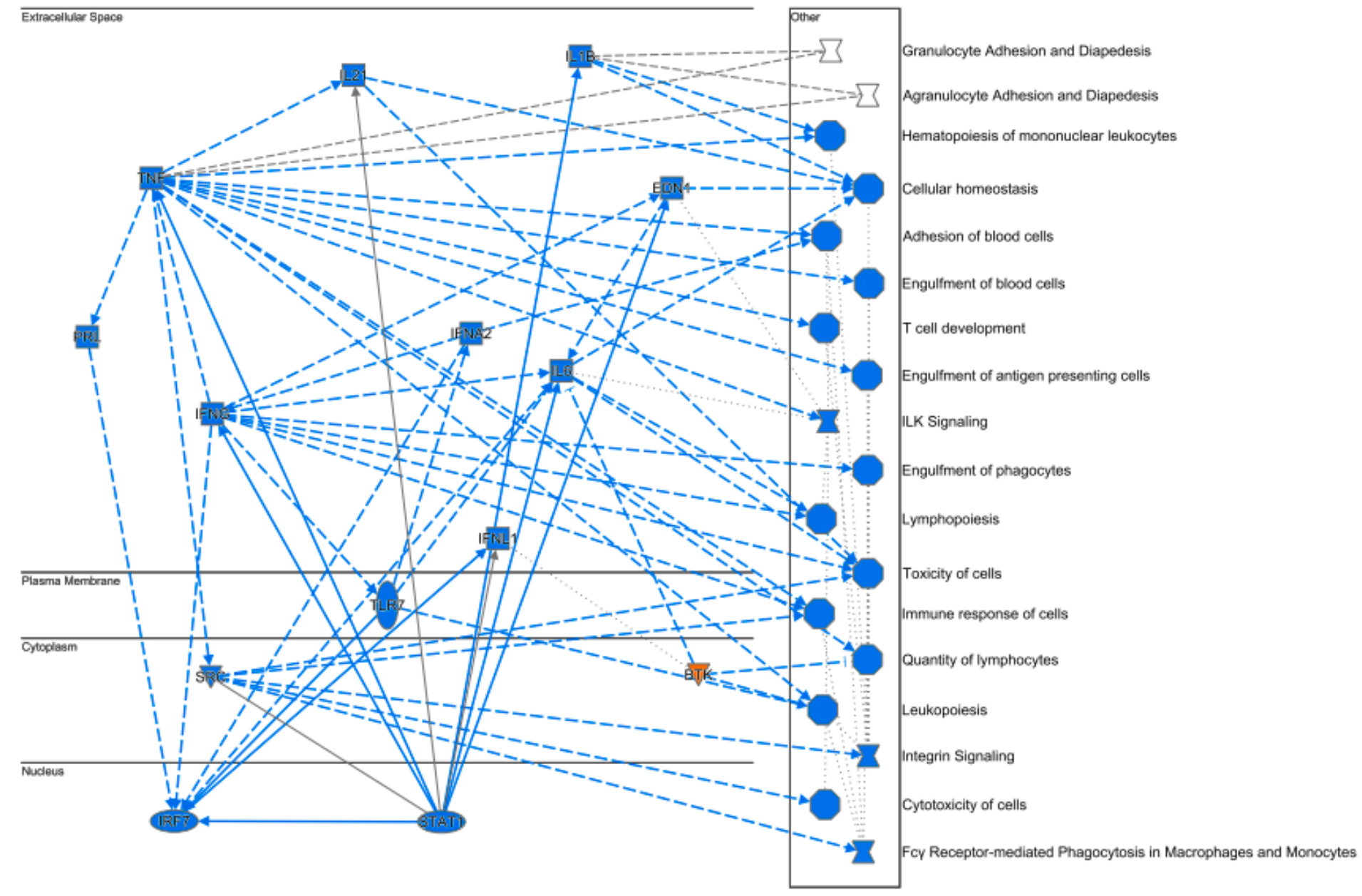

Figure 1

Graphical summary of effects of LMWF5A on LPS/IFNY-stimulated PBMC. Differential expression analysis comparing LMWF5A and saline treatment in LPS/IFNY-stimulated PBMC were uploaded into IPA software, and the graphical summary algorithm was run with the 'default node' and 'subcellular localization' settings to visualize the overall effects of LMWF5A treatment. For nodes and lines connecting nodes: blue $=$ inhibition, orange $=$ activation, gray $=$ directional effect not predicted, solid line $=$ direct interaction, dotted line $=$ indirect interaction . 


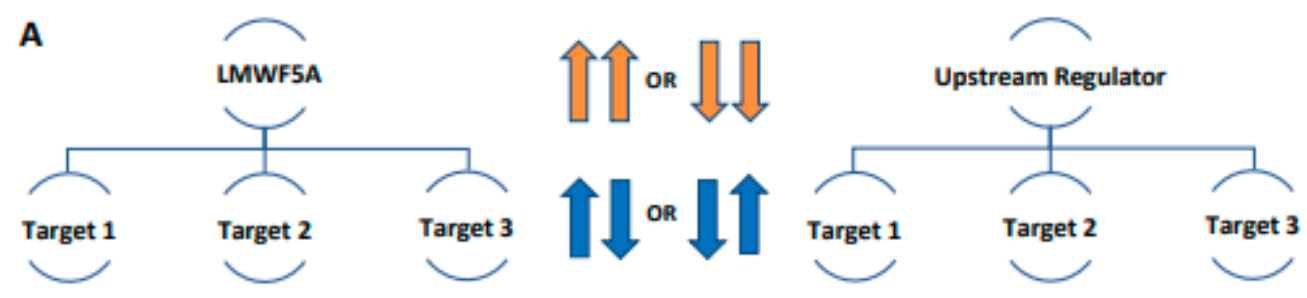

B

\begin{tabular}{|c|c|c|c|}
\hline upstream regulator & \multicolumn{1}{c}{ LPS } & \multicolumn{1}{c|}{ LPS/IFNY } & IL-4/IL-13 \\
\hline dexamethasone & 3.1 & 2.6 & 2.4 \\
\hline SIRT1 & 2.2 & 2.2 & 2.7 \\
\hline PTGER4 & 2.4 & 2.0 & 2.0 \\
\hline filgrastim & 1.5 & 1.3 & 3.2 \\
\hline SB203580 & 3.1 & 2.9 & N/A \\
\hline
\end{tabular}

C

\begin{tabular}{|c|c|c|c|}
\hline upstream regulator & \multicolumn{1}{|c|}{ LPS } & \multicolumn{1}{c|}{ LPS/FNY } & IL-4/L-13 \\
\hline lipopolysaccharide & -4.5 & -4.4 & -3.3 \\
\hline IFNG & -4.0 & -4.1 & -3.2 \\
\hline STAT1 & -4.1 & -3.1 & -2.9 \\
\hline poly rl::rC-RNA & -3.8 & -3.4 & -2.6 \\
\hline IFNA2 & -3.7 & -3.3 & -2.0 \\
\hline
\end{tabular}

\section{Figure 2}

Upstream regulator analysis. (a) Concept of upstream regulator analysis. Differential expression datasets for LMWF5A versus saline in PBMC stimulated with LPS, LPS/IFNy, or IL-4/IL-13 were individually subjected to upstream regulator analysis. IPA was utilized to compare the observed expression changes in the datasets that were due to LMWF5A with known directional changes of targets by upstream regulators found in the literature and comprising the IPA database. The identification of targets with correlated (either increased or decreased; orange) or inversely correlated (blue) directional changes allowed for statistical determination of similar or opposing activities between a particular upstream regulator and LMWF5A. (b-c) Results of upstream regulator analysis. The top five molecules that were identified to positively (b) or negatively (C) correlate with the activity of LMWF5A in the IPA upstream regulator analysis are listed. Statistical significance was determined by z-score, with a z-score of $>2$ representing a significant positive correlation and a z-score of <-2 representing a significant negative correlation. The tables are sorted by rows with the highest (b) or lowest (c) total z-score for all three stimulation conditions. 


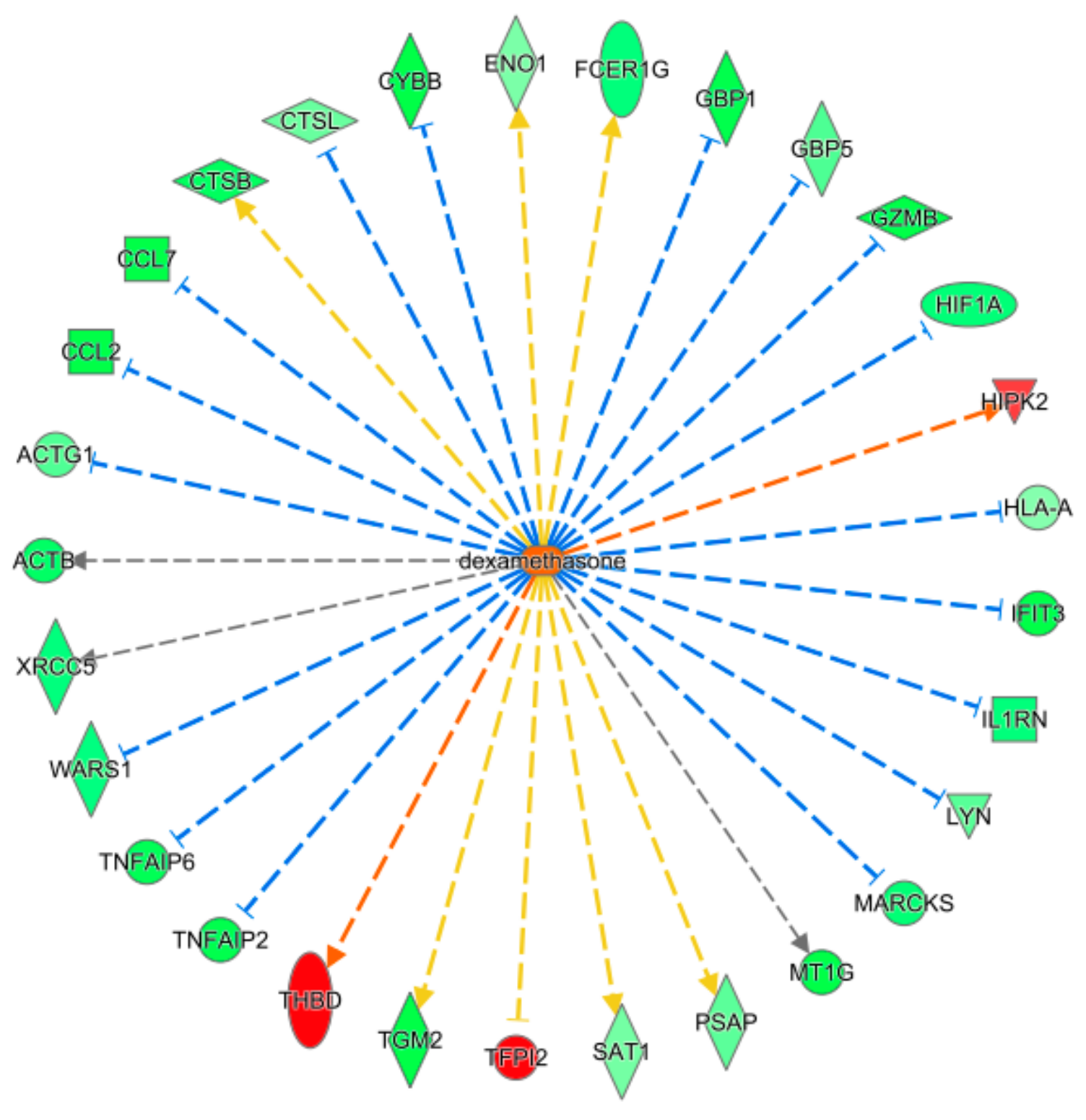

Figure 3

Common targets of dexamethasone and LMWF5A in LPS/IFNY-stimulated PBMC. Upstream regulator analysis was performed in IPA on differentially expressed genes due to LMWF5A treatment versus saline in LPS/IFNY-stimulated PBMC. Dexamethasone was selected and displayed as a network to visualize the 29 targets that are common to dexamethasone and LMWF5A. Decreased gene expression by LMWF5A (green); increased gene expression by LMWF5A (red); directionally consistent with dexamethasone (blue lines = inhibited; orange lines = activated); directionally inconsistent with dexamethasone (yellow lines); affected but direction unable to be predicted (gray lines). 


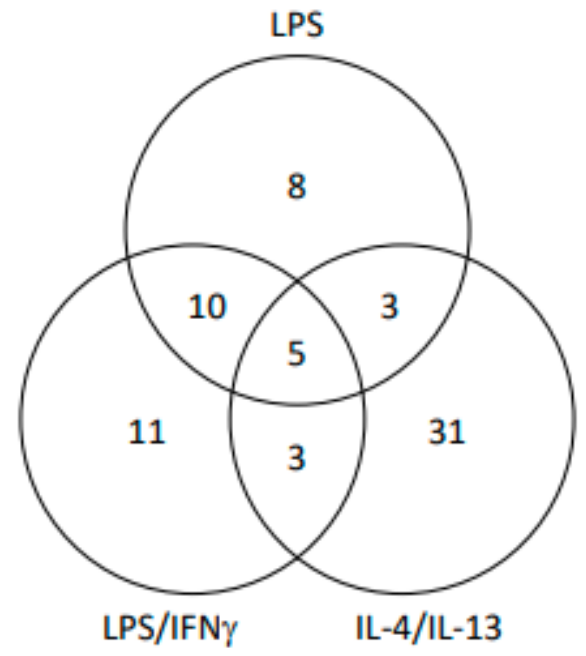

Figure 4

Comparison of PBMC stimulation conditions for LMWF5A/dexamethasone targets. Targets of LMWF5A and dexamethasone were identified by upstream regulator analysis in IPA as described above. A Venn diagram was constructed to depict the number of targets and their overlap for PBMC stimulated with LPS, LPS/IFNY, or IL-4/IL-13.

\section{Supplementary Files}

This is a list of supplementary files associated with this preprint. Click to download.

- Supp.Fig.1a.pdf

- Supp.Fig.1b.pdf

- Supp.Fig.2a.pdf

- Supp.Fig.2b.pdf 